\title{
On an new Open type variant of Newton's method
}

\author{
M. K. SINGH
}

\begin{abstract}
The aim of the present paper is to introduce and investigate a new Open type variant of Newton's method for solving nonlinear equations. The order of convergence of the proposed method is three. In addition to numerical tests verifying the theory, a comparison of the results for the proposed method and some of the existing ones have also been given.
\end{abstract}

Additional Key Words and Phrases: Newton's method, Iteration function, Order of convergence, Function evaluations, Efficiency index, Newton- Cotes formula.

\section{INTRODUCTION}

In reliability In Science and Engineering, many of the nonlinear and transcendental problems, of the form $=0$, are complex in nature. Since it is not always possible to obtain its exact solution by usual algebraic process, therefore numerical iterative methods such as Newton, secant methods are often used to obtain the approximate solution of such problems. Many optimization problems also lead to such equations, since to solve the nonlinear equation problem is equivalent to minimizing a master function described as follows

$$
\left\{\begin{array}{c}
\text { Find: } x, x \in \varphi \\
\operatorname{Min} F(x)=f^{2}(x)
\end{array}\right.
$$

Where $\varphi$ is the solution space. This master function is positive definite and has a global minimum at each of the roots. When the minimization of $F(x)$ is 0 , the corresponding is the exact solution.

Now considering the problem of finding a real zero of a function $f: R \rightarrow R$. This zero can be determined as a fixed point of some iteration function $g$ by means of the one-point iteration method

$$
x_{n+1}=g\left(x_{n}\right) \quad n=0,1, \ldots
$$


Where $x_{0}$ is the starting value. The best known and the most widely used example of these types of methods is the classical Newton's method [8] given by

$$
x_{n+1}=x_{n}-\frac{f\left(x_{n}\right)}{f^{\prime}\left(x_{n}\right)} \quad n=0,1, \ldots
$$

Given a starting point $x_{0}$ in Newton' method, a root of Eq. (1) is found as the limit of a sequence of $\left\{x_{n}\right\}$. Each update $x_{n+1}$ is found as the unique zero of the tangent line to the curve $y=f(x)$ at $x_{n}$ provided $f^{\prime}\left(x_{n}\right) \neq 0$. If $x_{0}$ is close enough to the solution $\alpha$ of (1) with $f^{\prime}(\alpha) \neq 0$, then Newton sequence $\left\{\left(x_{n}\right)\right\}$

$$
x_{n+1}=x_{n}-\frac{f\left(x_{n}\right)}{f^{\prime}\left(x_{n}\right)} \quad n=0,1, \ldots
$$

is well defined and converges to $\alpha$, and the convergence is at least quadratic if $f^{\prime \prime}(\alpha)$ is continuous near $\alpha$. It converges quadratic ally to simple zeros and linearly to multiple zeros. In the literature, its several modifications [1,2,3,5] have been introduced in order to accelerate it or to get a method with a higher order of convergence at the expense of additional evaluations of functions, derivatives and changes in the points of iterations.

\section{DEFINITIONS}

DEFINITION 2.1. (See [4] [6]) - Classifying the iteration function by the information they require, let $x_{n+1}$ be determined only by information at $x_{n}$. No old information is reused. Thus

$$
x_{n+1}=g\left(x_{n}\right) \quad n=0,1,2 \ldots
$$

Then $\mathrm{g}$ is called on point iteration function, the most commonly known example of a one point iteration function is Newton iteration function. 
DEFINITION 2.2. (See [4] [6]) -

Let $x_{n+1}$ be determined by information at $x_{n}$ and reused information at $x_{n-1,} x_{n-2, \cdots, x_{n-N}}$.

Thus

$$
x_{n+1}=g\left(x_{n} ; x_{n-1}, \ldots, x_{n-N}\right) \quad n=0,1,2 \ldots
$$

Then $\mathrm{g}$ is called one point iteration function with memory. The semi colon separates the point at which the new data are used.

The best known example of one point iteration function with memory is secant iteration function.

DEFINITION 2.3. (See [4] [6]) -

Let $x_{n+1}$ be determined by new information at $x_{n}$ and reused information at $w_{1}\left(x_{n}\right), \ldots, w_{n}\left(x_{n}\right)$. No old information is reused. Thus

$$
x_{n+1}=g\left(x_{n}, w_{1}\left(x_{n}\right), \ldots, w_{n}\left(x_{n}\right) . .\right) \quad n=0,1,2 \ldots
$$

Then $\mathrm{g}$ is called multi - point iteration function.

DEFINITION 2.4. (See [4] [6]) -

Let $x_{n+1}$ be determined by new information at $v_{n}$ and reused information at $v_{n-1,} v_{n-2, \cdots, v_{n-N}}$. No old information is reused. Thus

$$
x_{n+1}=g\left(v_{n} ; v_{n-1}, \ldots, n_{n-N}\right) \quad n=0,1,2 \ldots
$$

Where $v_{n}$ represents $x_{n}, w_{1}\left(x_{n}\right), \ldots, w_{n}\left(x_{n}\right) \quad n=0,1,2, \ldots$

Then $\mathrm{g}$ is called multi - point iteration function with memory.

DEFINITION 2.5. Considering the problem of numerical approximation of a real roots $\alpha$ of the non linear equation

$$
f(x)=0, \quad f: D \subseteq R \rightarrow R
$$


The root $\alpha$ is said to be simple if $f(\alpha)=0$ and $f^{\prime}(\alpha) \neq 0$. If $f(\alpha)=f^{\prime}(\alpha)=\ldots=f^{m-1}(\alpha)=0$ and $f^{m}(\alpha) \neq 0$ for $m \geq 1$ then $\alpha$ is of multiplicity $m$.

DEFINITION 2.6. (See [6]) - If the sequence $\left\{x_{n} / n \geq 0\right\}$ tends to a limit $\alpha$ in such a way that

$$
\lim _{x_{n} \rightarrow \alpha} \frac{x_{n+1}-\alpha}{\left(x_{n}-\alpha\right)^{p}}=C=\left|g^{(p)}(\alpha)\right| / p
$$

For $C \neq 0$ some and $p \geq 1$, then the order of convergence of the sequence is said to be $p$, and $C$ is known as asymptotic error constant.

When $p=1$, the convergence is linear, and it is called first order convergence.

While for $p=2$ and $p=3$ the sequence is said to converge quadratically and cubically, respectively.

The value of $p$ is called the order of convergence of the method which produces the sequence $\left\{x_{n} / n \geq 0\right\}$. Let $e_{n}=x_{n}-\alpha$ Then the relation $e_{n+1}=C e_{n}{ }^{p}+O\left(e_{n}{ }^{p+1}\right)$ is called the error equation for the method, $p$ being the order of convergence.

DEFINITION 2.7. (See [4,7])

Efficiency index is simply defined as $p^{1 / m}$ where $p$ is the order of the method and $m$ is the number of functions evaluations required by the method (units of work per iteration). Then the efficiency index of Newton's method is 1.414 and iterative methods with order of convergence three and taking three functions evaluations has efficiency index 1.442 .

DEFINITION 2.8. (See [4, 7]) - For any iterative method (1), if the order is the same for zeros of all multiplicities, we say that the order is the multiplicity independent. If the order depends on the multiplicity, we say that the order is the multiplicity dependent. 
DEFINITION 2.9. (See [4,7]) - There are two types of Newton-Cotes formulae, the "closed" type which uses the function value at all points, and the "open" type which does not use the function values at the endpoints.

\section{DESCRIPTION OF THE METHODS}

Let $\alpha$ be a simple zero of a sufficiently differentiable function $f$ and consider the numerical solution of the equation $f(x)=0$, then it can be written as - see[1]

$$
f(x)=f\left(x_{n}\right)+\int_{x_{n}}^{x} f^{\prime}(t) d t
$$

Suppose, we interpolate $f^{\prime}$ on the interval $\left[x_{n}, x\right]$ by constant $f^{\prime}\left(x_{n}\right)$, then $\left(x-x_{n}\right) f^{\prime}\left(x_{n}\right)$ provides an approximation for the integral in (9) and by taking $x=\alpha$ we obtain

$$
0 \approx f\left(x_{n}\right)+\left(\alpha-x_{n}\right) f^{\prime}\left(x_{n}\right)
$$

And hence, a new approximation $x_{n+1}$ to $\alpha$ is given by

$$
x_{n+1}=x_{n}-\frac{f\left(x_{n}\right)}{f^{\prime}\left(x_{n}\right)} \quad n=0,1, \ldots
$$

Which is Newton's method for $n=0,1, \ldots$. On the other hand, if we approximate the indefinite integral in (9) by the trapezoidal rule and take $x=\alpha$, we obtain

$$
0 \approx f\left(x_{n}\right)+1 / 2\left(\alpha-x_{n}\right)\left(f^{\prime}\left(x_{n}\right)+f^{\prime}(\alpha)\right)
$$

And therefore, a new approximation $x_{n+1}$ to $\alpha$ is given by

$$
x_{n+1}=x_{n}-\frac{2 f\left(x_{n}\right)}{f^{\prime}\left(x_{n}\right)+f^{\prime}\left(x_{n+1}\right)}
$$

If the $(n+1)^{t h}$ value of Newton's method is used on the right-hand side of the above equation to overcome the implicity problem, then

$$
x_{n+1}=x_{n}-\frac{2 f\left(x_{n}\right)}{f^{\prime}\left(x_{n}\right)+f^{\prime}\left(z_{n+1}\right)} \quad, \text { where } z_{n+1}=x_{n}-\frac{f\left(x_{n}\right)}{f^{\prime}\left(x_{n}\right)}
$$


is obtained which is, for $n=0,1, \ldots$, the trapezoidal Newton's method of Weerakoon [1].

Let us rewrite equation (10) as

$$
x_{n+1}=x_{n}-\frac{f\left(x_{n}\right)}{\left(f^{\prime}\left(x_{n}\right)+f^{\prime}\left(z_{n+1}\right)\right) / 2} \quad, n=0,1,2, \ldots
$$

And further to re-write as -

$$
z_{n}=x_{n}-\frac{f\left(x_{n}\right)}{\left(f^{\prime}\left(x_{n}\right)+f^{\prime}\left(y_{n}\right)\right) / 2} \quad, n=0,1,2, \ldots
$$

So this variant of Newton's method can be viewed as obtained by using arithmetic mean of $f^{\prime}\left(x_{n}\right)$ and $f^{\prime}\left(y_{n}\right)$ instead of $f^{\prime}\left(x_{n}\right)$ in Newton s method de ned by (1), which was called as arithmetic mean Newton's method.

\section{New variant of Newton method -}

In (9) if we use the open type quadrature formula for $n=3$

$$
=\int_{a}^{b} f(x) d x=\sum_{k=1}^{n} f\left(x_{k}\right)
$$

For $n=3 \mid=\frac{3 h}{2}[f(a+h)+f(a+2 h)]$, error term $R_{3}=\frac{3}{4} h^{3} f^{\prime \prime}(\xi)$

$$
\frac{b-a}{2}\left[f\left(\frac{2 a+b}{3}\right)+f\left(\frac{a+2 b}{3}\right)\right]
$$

From Newton's theorem

$$
f(x)=f\left(x_{n}\right)+\int_{x_{n}}^{x} f^{\prime}(t) d t
$$

Using the above formula (12)

$$
x_{n+1}=x_{n}-\frac{2 f\left(x_{n}\right)}{f^{\prime}\left(\frac{2 x_{n}+\alpha}{3}\right)+f^{\prime}\left(\frac{x_{n}+2 \alpha}{3}\right)}
$$


Now a new approximation $x_{n+1}$ to $\alpha$ is given by

$$
x_{n+1}=x_{n}-\frac{2 f\left(x_{n}\right)}{f^{\prime}\left(\frac{2 x_{n}+z}{3}\right)+f^{\prime}\left(\frac{x_{n}+2 z}{3}\right)} \quad \text { where } z=x_{n}-\frac{f\left(x_{n}\right)}{f^{\prime}\left(x_{n}\right)}
$$

Which we called as Open type Newton's method (ONM) . Classifying the iteration function by the information they require, we conclude that it is a Multi point iteration function (two point iteration function).

\section{CONVERGENCE ANALYSIS}

THEOREM 4.1. Let $\alpha \varepsilon$ I be a simple zero of a sufficiently differentiable function $f: I C R \rightarrow R$ for an open interval I. If $x_{0}$ is sufficiently close to $\alpha$, then the methods defined by (13) converges cubically.

PROOF. Let $\alpha \varepsilon$ I be a simple zero of $f$. Since $f$ is sufficiently differentiable, by expanding $f\left(x_{n}\right)$ and $f^{\prime}\left(x_{n}\right)$ about $\alpha$ we get

$$
\begin{aligned}
& f\left(x_{n}\right)=f\left(\alpha+e_{n}\right)=f^{\prime}(\alpha) e_{n}+f^{\prime \prime}(\alpha) \frac{e_{n}^{2}}{! 2} f^{\prime \prime}(\alpha) \frac{e_{n}^{3}}{! 3}+O\left(e_{n}^{4}\right)= \\
& =f^{\prime}(\alpha)\left[e_{n}+c_{2} e_{n}{ }^{2}+c_{3} e_{n}^{3}+O\left(e_{n}^{4}\right)\right]
\end{aligned}
$$

Where $C_{K}=(1 / ! k) f^{k}(\alpha) / f^{\prime}(\alpha)$

$$
\begin{aligned}
& f^{\prime}\left(x_{n}\right)=f^{\prime}\left(\alpha+e_{n}\right)=f^{\prime}(\alpha) e_{n}+f^{\prime \prime}(\alpha) e_{n}+f^{\prime \prime}(\alpha) \frac{e_{n}^{2}}{! 3}+O\left(e_{n}^{4}\right)= \\
& =f^{\prime}(\alpha)\left[1+2 C_{2} e_{n}+3 C_{3} e_{n}^{2}+4 C_{4} e_{n}^{3}+O\left(e_{n}^{4}\right)\right] \\
& \frac{f\left(x_{n}\right)}{f^{\prime}\left(x_{n}\right)}=\left[e_{n}+C_{2} e_{n}{ }^{2}+C_{3} e_{n}^{3}+O\left(e_{n}^{4}\right)\right]\left[1+2 C_{2} e_{n}+3 C_{3} e_{n}{ }^{2}+4 C_{4} e_{n}^{3}+O\left(e_{n}^{4}\right)\right]^{-1} \\
& =\left[e_{n}+C_{2} e_{n}{ }^{2}+\left(2 C_{2}-2 C_{3}\right) e_{n}^{3}+O\left(e_{n}^{4}\right)\right]
\end{aligned}
$$


Newton's method is

$$
\begin{aligned}
& x_{n+1}=x_{n}-\frac{f\left(x_{n}\right)}{f^{\prime}\left(x_{n}\right)} \quad n=0,1, \ldots \\
& e_{n+1}=C_{2} e_{n}^{2}+\left(2 C_{3}-2 C_{2}^{2}\right) e_{n}^{3}+O\left(e_{n}^{4}\right)
\end{aligned}
$$

That is Newton's method converges quadratic ally

and hence, for $z$ given in (4) we have

$$
\begin{gathered}
z=\alpha+C_{2} e_{n}{ }^{2}+\left(2 C_{3}-2 C_{2}{ }^{2}\right) e_{n}^{3}+O\left(e_{n}{ }^{4}\right) \\
x_{n+1}=x_{n}-\frac{2 f\left(x_{n}\right)}{f^{\prime}\left(\frac{2 x_{n}+z}{3}\right)+f^{\prime}\left(\frac{x_{n}+2 z}{3}\right)} \quad \text { where } z=x_{n}-\frac{f\left(x_{n}\right)}{f^{\prime}\left(x_{n}\right)} \\
\left(\frac{2 x_{n}+z}{3}\right)=\frac{2 \alpha+2 e_{n}+\alpha+C_{2} e_{n}{ }^{2}+\left(2 C_{3}-2 C_{2}{ }^{2}\right) e_{n}{ }^{3}+O\left(e_{n}{ }^{4}\right)}{3}= \\
=\frac{3 \alpha+2 e_{n}+C_{2} e_{n}{ }^{2}+2\left(C_{3}-C_{2}{ }^{2}\right) e_{n}{ }^{3}+O\left(e_{n}{ }^{4}\right)}{3} \\
f^{\prime}\left(\frac{2 x_{n}+z}{3}\right)=f^{\prime}\left(\alpha+\frac{2}{3} e_{n}+\frac{C_{2}}{3} e_{n}{ }^{2}+\frac{2}{3}\left(C_{3}-C_{2}{ }^{2}\right) e_{n}{ }^{3}+O\left(e_{n}{ }^{4}\right)\right)
\end{gathered}
$$

On simplifications we get

$$
f^{\prime}(\alpha)\left(1+\frac{4}{3} C_{2} e_{n}+\left(\frac{2}{3} C_{2}^{2}+\frac{4}{3} C_{3}\right) e_{n}^{2}+\left\{\frac{8}{3} C_{2} C_{3}-\frac{4}{3} C_{2}^{3}+\frac{32}{27} C_{4}\right\} e_{n}^{3}+O\left(e_{n}^{4}\right)\right)
$$

Again

$$
\begin{aligned}
& \left(\frac{x_{n}+2 z}{3}\right)=\frac{\alpha+e_{n}+2 \alpha+2 C_{2} e_{n}^{2}+4\left(C_{3}-C_{2}^{2}\right) e_{n}^{3}+O\left(e_{n}^{4}\right)}{3} \\
& f\left(\frac{x_{n}+2 z}{3}\right)=f^{\prime}(\alpha)\left(1+\frac{2}{3} C_{2} e_{n}+\left(\frac{4}{3} C_{2}^{2}+\frac{1}{3} C_{3}\right) e_{n}^{2}+\left\{4 C_{2} C_{3}-\frac{8}{3} C_{2}^{3}+\frac{4}{9} C_{4}\right\} e_{n}^{3}+O\left(e_{n}^{4}\right)\right)
\end{aligned}
$$


Adding (12) and (13) we have

$$
\begin{aligned}
& f\left(\frac{2 x_{n}+z}{3}\right)+f\left(\frac{x_{n}+2 z}{3}\right) \\
& f^{\prime}(\alpha)\left(1+\frac{4}{3} C_{2} e_{n}+\left(\frac{2}{3} C_{2}{ }^{2}+\frac{4}{3} C_{3}\right) e_{n}{ }^{2}+\left\{\frac{8}{3} C_{2} C_{3}-\frac{4}{3} C_{2}{ }^{3}+\frac{32}{27} C_{4}\right\} e_{n}^{3}+O\left(e_{n}^{4}\right)\right) \\
& + \\
& f^{\prime}(\alpha)\left(1+\frac{2}{3} C_{2} e_{n}+\left(\frac{4}{3} C_{2}{ }^{2}+\frac{1}{3} C_{3}\right) e_{n}{ }^{2}+\left\{4 C_{2} C_{3}-\frac{8}{3} C_{2}^{3}+\frac{4}{9} C_{4}\right\} e_{n}^{3}+O\left(e_{n}^{4}\right)\right) \\
& =2 f^{\prime}(\alpha)\left(1+C_{2} e_{n}+\left(C_{2}{ }^{2}+\frac{5}{6} C_{3}\right) e_{n}{ }^{2}+\left\{\frac{10}{3} C_{2} C_{3}-2 C_{2}{ }^{3}+\frac{22}{27} C_{4}\right\} e_{n}^{3}+O\left(e_{n}^{4}\right)\right) \\
& =x_{n}-\frac{2 f\left(x_{n}\right)}{f^{\prime}\left(\frac{2 x_{n}+z}{3}\right)+f^{\prime}\left(\frac{x_{n}+2 z}{3}\right)} \quad \text { where } \quad z=x_{n}-\frac{f\left(x_{n}\right)}{f^{\prime}\left(x_{n}\right)} \\
& =\frac{2 f^{\prime}(\alpha)\left[e_{n}+C_{2} e_{n}^{2}+C_{3} e_{n}^{3}+O\left(e_{n}^{4}\right)\right]}{2 f^{\prime}(\alpha)\left(1+C_{2} e_{n}+\left(C_{2}^{2}+\frac{5}{6} C_{3}\right) e_{n}^{2}+\left\{\frac{10}{3} C_{2} C_{3}-2 C_{2}^{3}+\frac{22}{27} C_{4}\right\} e_{n}^{3}+O\left(e_{n}^{4}\right)\right)} \\
& =\left[e_{n}+C_{2} e_{n}^{2}+C_{3} e_{n}^{3}+O\left(e_{n}^{4}\right)\right]\left(1+C_{2} e_{n}+\left(C_{2}^{2}+\frac{5}{6} C_{3}\right) e_{n}^{2}+\left\{\frac{10}{3} C_{2} C_{3}-2 C_{2}^{3}+\frac{22}{27} C_{4}\right\} e_{n}^{3}+O\left(e_{n}^{4}\right)\right)^{-1} \\
& =\left[e_{n}+C_{2} e_{n}^{2}+C_{3} e_{n}^{3}+O\left(e_{n}^{4}\right)\right]\left(1-C_{2} e_{n}-\frac{5}{6} C_{3} e_{n}^{2}+\left\{-\frac{5}{3} C_{2} C_{3}+5 C_{2}^{3}-\frac{22}{27} C_{4}\right\} e_{n}^{3}+O\left(e_{n}^{4}\right)\right)= \\
& =\left[e_{n}+\left(-\frac{5}{6} C_{3}-C_{2}^{2}+C_{3}\right) e_{n}^{3}+O\left(e_{n}^{4}\right)\right] \\
& =\left[e_{n}+\left(-C_{2}^{2}+\frac{1}{6} C_{3}\right) e_{n}^{3}+O\left(e_{n}^{4}\right)\right]
\end{aligned}
$$


Open type Newton formula is

$$
\begin{aligned}
& x_{n+1}=x_{n}-\frac{2 f\left(x_{n}\right)}{f^{\prime}\left(\frac{2 x_{n}+z}{3}\right)+f^{\prime}\left(\frac{x_{n}+2 z}{3}\right)} \quad \text { where } z=x_{n}-\frac{f\left(x_{n}\right)}{f^{\prime}\left(x_{n}\right)} \\
& \alpha+e_{n+1}=\left[\alpha+e_{n}-e_{n}-\left(-C_{2}^{2}+\frac{1}{6} C_{3}\right) e_{n}^{3}+O\left(e_{n}^{4}\right)\right] \\
& e_{n+1}=\left[\left(C_{2}{ }^{2}-\frac{1}{6} C_{3}\right) e_{n}{ }^{3}+O\left(e_{n}^{4}\right)\right]
\end{aligned}
$$

Which shows that new open type Newton's method (ONM) converges cubically.

\section{NUMERICAL RESULTS AND CONCLUSION}

In this section, we present the results of some numerical tests to compare the efficiencies of the methods. We employed Classical Newton's Method, Arithmetic mean Newton's method of Fernando et al. [i], and Geometric mean Newton's method and Open type Newton's method (ONM) that we developed. Numerical computations reported here have been carried out in MATLAB. The stopping criterion has been taken as $\left|x_{n+1}-\alpha\right|+\left|f\left(x_{n+1}\right)\right|<10^{-14}$.

EXAMPLE 1. Consider the equation $f(x)=x^{3}+4 x^{2}-10$

We start with $x_{n}=-0,2$. Newton's iteration take 17 iterations, Arithmetic mean Newton's method takes 15 iterations, and Proposed Open type Newton's method takes only 5 iterations in order to find the exact root $\alpha=1.365230013414097$.

EXAMPLE 2. Consider the equation $f(x)=\cos x-x$

We start with $x_{n}=3$. Newton's iteration take 6 iterations, Arithmetic mean Newton's method takes 9 iterations, Geometric mean Newton's method Fails and Proposed Open type Newton's method takes only 5 iterations in order to find the exact $\operatorname{root} \alpha=0.7390851332151607$. 
EXAMPLE 3. Consider the equation $f(x)=x^{3}-e^{-x}$

We start with $x_{n}=2$. Newton's iteration take 7 iterations, Arithmetic mean Newton's method takes 4 iterations, Geometric mean Newton's method takes 25 iterations and Proposed Open type Newton's method takes only 4 iterations in order to find the exact root $\alpha=0.772882959149210$.

Here we have studied the properties and definitions related to open type Newton method further The convergence analysis of the method is performed in much simpler way to show that the convergence order of the method is three .

All numerical results are in accordance with the theory and the basic advantage of the variants of Newton's method based on means or integration methods that they do not require the computation of second- or higher-order derivatives The main characteristic of the ONM are : at least third order of convergence for the simple roots, at least linear order of convergence for the multiple roots.

As far as the numerical results are considered, Open type Newton method ONM gives more accurate result and can be compared with Newton Method, and other third order variants of Newton's method.

\section{REFERENCES}

[1] S. Weerakoon and T.G.I. Fernando, A variant of Newton's method with accelerated third- order convergence, Appl. Math. Lett. 13 (8), 87-93, (2000).

[2] W.F. Ford and J.A. Pennline, Accelerated convergence in Newton's method, SIAM Review 38, 658659,(1996).

[3] J. Gerlach, Accelerated convergence in Newton's method, SIAM Review 36, 272-276, (1994).

[4] R. Wait, the Numerical Solution of Algebraic Equations, John Wiley \& Sons, (1979).

[5] M. Igarashi, A termination criterion for iterative methods used to find the zeros of polynomials, Math. Comp. 42, 165-171, (1984).

[6] A.Y.Ozban, Some new variants of Newton's method, Applied Mathematics Letters 17, (2004), 677- 682

[7] J.F. Traub, Iterative Methods for the Solution of Equations, Prentice Hall, Clifford, NJ, 1964.

\section{Manoj Kumar Singh}

Department of Mathematics, Faculty of science

Banaras Hindu University

Varanasi, India 